\title{
New General Formulation and Experimental Verification of Harmonic Clipping Contours in High-Frequency Power Devices
}

\author{
Roberto Quaglia, Member, IEEE, James J. Bell, and Steve Cripps, Life Fellow, IEEE
}

\begin{abstract}
This paper presents a novel closed-form solution for the theoretical calculation of harmonic clipping contours when an arbitrary number of harmonics is considered. The clipping contours can be used to design the loads of a high-frequency power device in order to avoid drain current clipping, and hence preventing strong nonlinear effects. For the first time the predicted second harmonic contours are validated thoroughly by means of experimental characterization of GaN HEMT devices. The measured contours result in good agreement with the theory. Moreover, the effect of third harmonic load tuning is also assessed and verified for the first time. These results prove that the clipping contours can be used as a tool for the systematic design of low-distortion power amplifiers.
\end{abstract}

Index Terms-Field-effect transistors, high-efficiency amplifiers, power amplifiers (PAs).

\section{INTRODUCTION}

$\mathbf{T}$ HE evolution of wireless digital communications has increased the need for high-frequency power amplifiers (PAs) characterized by low distortion. At the same time, maximizing the PA efficiency is another critical design target, since the PA is one of the most power hungry components in a wireless transmitter.

Once the active device is chosen according to several considerations, e.g., frequency band, output power, or cost, then the bias point and the harmonic terminations can be tuned to optimize the tradeoff between efficiency, output power, and linearity. The class AB PA is probably the most common example of an efficient and linear PA, and relies on a purely resistive fundamental load and short-circuited higher harmonics. However, other solutions have been proposed in the literature to improve PA performance by suitable design of harmonic terminations [1]-[6]. The work in [7] demonstrated that class $\mathrm{B}$ (or class $\mathrm{AB}$ ) is in fact a particular case in a continuum of high-efficiency linear modes in PAs, called the $\mathrm{B} / \mathrm{J}$ continuum, all characterized as having the same maximum output power and efficiency. This "design space" can be exploited to design wideband PAs, overcoming the purely resistive fundamental load assumption of class $B$ that can be difficult to achieve in high-frequency PAs due to the device's

Manuscript received December 12, 2016; revised February 13, 2017 accepted March 20, 2017. Date of publication May 9, 2017; date of current version October 4, 2017. This project was supported by the European Union's Horizon 2020 Research and Innovation Programme under Marie SkłodowskaCurie Grant 654987. (Corresponding author: Roberto Quaglia.)

The authors are with the Centre for High Frequency Engineering, Cardiff University, Cardiff CF24 3AA, U.K. (e-mail: quagliar@cardiff.ac.uk).

Color versions of one or more of the figures in this paper are available online at http://ieeexplore.ieee.org.

Digital Object Identifier 10.1109/TMTT.2017.2687900 reactive elements. To allow for this relaxation of fundamental loading condition, the second harmonic load must be moved from a short circuit to specific reactive loads in order to avoid knee voltage violation. Other classes of continuous modes have since evolved, based on higher harmonics or different device operation [8]-[11].

The work in [12] identified a possible issue when designing a continuous mode PA: in broadband design it becomes difficult to maintain the second harmonic on a purely reactive load frequency trajectory. This nonideal load condition, if not properly dealt with, can cause waveform clipping and, as a consequence, high distortion. Experimental data showed that it is possible to identify second harmonic contours that separate the "clipping" loads from the "non-clipping" loads, and that the loads lying on this contour are leading in fact to a drain voltage waveform that "grazes" the knee voltage.

The introduced clipping contours can be used as a powerful tool to compromise systematically the power/efficiency performance (determined by the fundamental load), the linearity, and the feasibility of the matching networks in a broadband PA design. A mathematical formulation of the clipping contours, in the case of fundamental and second harmonic, has been given in [13]. In particular, it is shown that, by reducing the intrinsic fundamental load below the nominal optimum $R_{\text {opt }}$, the second harmonic clipping contour enters the passive Smith Chart domain, thus allowing for the use of nonpurely reactive second harmonic loads while maintaining an unclipped waveform. Another formulation of the clipping contours for fundamental and second harmonic has been introduced in [14], and described as a resistive/reactive class $\mathrm{J}$ mode.

In this paper, a new formulation for the clipping contours is introduced, which also allows for the extension to an arbitrary number of harmonics. In particular, it is shown how the third harmonic can be used to move clipping contours, confirming the results of [15], where a numerical method was used to calculate higher harmonics clipping contours. The previous work in [13] provided an experimental observation of the effects of violating clipping contours through the linearity measurements on a prototype PA. In this paper, for the first time, the clipping contours are experimentally verified by means of systematic harmonic source- and load-pull measurements, including the validation of the beneficial effects of third harmonic load tuning. The latter step is of great importance from a PA designer's perspective because it validates a condition that permits an increase to the second harmonic load "design space" without reducing the output power. This methodology is applicable to linear PAs, while it might not be suitable where 
significant compression levels must be tolerated as in saturated PAs for very high efficiency.

This paper is organized as follows. Section II shows the new formulation of the clipping contours, for the second harmonic and for an arbitrary number of harmonics. Section III describes the experimental characterization campaign, comparing the measurement results with the proposed theory. Finally, in Section IV some conclusions are drawn.

\section{Closed-Form Solution}

Clipping contours are defined as the region of loads, at the $k$ th harmonic, which provide a zero-grazing voltage waveform for given input drive and other specified harmonic loads. In a real device, zero can be substituted by $V_{\min }$, a value related to the knee voltage, which represents the boundary between the quasi-linear and the strong nonlinear behavior of the device. To simplify the representation, the voltage waveform can be normalized by $V_{0}=V_{\mathrm{DD}}-V_{\mathrm{min}}$, where $V_{\mathrm{DD}}$ is the drain bias voltage. By setting the normalized bias at 1 , a zero-grazing condition can still be considered.

Drain current can be expressed in terms of Fourier series. Assuming unilateral device behavior, with constant-piecewise transconductance, the fixed input drive translates into a rectified sinusoidal drain current waveform with constant conduction angle, whose Fourier components can be calculated in closed form [16]. In particular, only cosine terms will be present, and the current can be expressed as

$$
I(\theta)=I_{0}+\sum_{m=1}^{\infty} I_{m} \cos (m \theta) .
$$

Since all current harmonic components are known, all but the $k$ th harmonic voltage components are known. As a consequence, the clipping contours can be derived by finding the family of voltage waveforms that graze zero and contain the known harmonic components. The approach used in [13] for the definition of this voltage waveform family is based on the factorization of the voltage waveform, typical of continuous modes. In this paper, a new formulation is proposed that can be extended to higher harmonics.

\section{A. New Formulation of Second Harmonic Clipping Contours}

The normalized voltage waveform can be written as

$$
\begin{aligned}
v(\theta)= & 1-v_{1 r} \cos (\theta)-v_{1 q} \sin (\theta)+ \\
& -v_{2 r} \cos (2 \theta)-v_{2 q} \sin (2 \theta) .
\end{aligned}
$$

The definition of zero-grazing condition is mathematically expressed as [7]

$$
\left\{\begin{array}{l}
v(\theta)=0 \\
d v(\theta) / d \theta=0
\end{array}\right.
$$

that deriving (2) becomes

$$
\left\{\begin{array}{l}
1-v_{1 r} \cos (\theta)-v_{1 q} \sin (\theta)+ \\
\quad-v_{2 r} \cos (2 \theta)-v_{2 q} \sin (2 \theta)=0 \\
v_{1 r} \sin (\theta)-v_{1 q} \cos (\theta) \\
\quad+2 v_{2 r} \sin (2 \theta)-2 v_{2 q} \cos (2 \theta)=0 .
\end{array}\right.
$$

The angle $\theta$ can be substituted by an independent variable $\phi$ swept in the range $[-\pi ; \pi[$. If either the fundamental or the second harmonic voltage is known, then the second or fundamental voltage can be derived as the system solution. A solution will be found for each $\phi$, and will correspond to a $v(\theta)$ with a minimum in $\theta=\phi$.

For a known second harmonic load, $v_{2 r}, v_{2 q}$ are known, and the system of (4) can be rewritten as

$$
\left\{\begin{array}{l}
v_{1 r} \cos (\phi)+v_{1 q} \sin (\phi)=1-A \\
v_{1 r} \sin (\phi)-v_{1 q} \cos (\phi)=B
\end{array}\right.
$$

where

$$
\begin{aligned}
& A=v_{2 r} \cos (2 \phi)+v_{2 q} \sin (2 \phi) \\
& B=2\left(-v_{2 r} \sin (2 \phi)+v_{2 q} \cos (2 \phi)\right) .
\end{aligned}
$$

With some simple calculations, the system is solved as

$$
\left\{\begin{array}{l}
v_{1 r}=(1-A) \cos (\phi)+B \sin (\phi) \\
v_{1 q}=(1-A) \sin (\phi)-B \cos (\phi) .
\end{array}\right.
$$

On the other hand, for a known fundamental load, $v_{1 r}$ and $v_{1 q}$ are known, and the system is solved as

$$
\left\{\begin{array}{l}
v_{2 r}=(1-A) \cos (2 \phi)+B \sin (2 \phi) \\
v_{2 q}=(1-A) \sin (2 \phi)-B \cos (2 \phi)
\end{array}\right.
$$

where

$$
\left\{\begin{array}{l}
A=v_{1 r} \cos (\phi)+v_{1 q} \sin (\phi) \\
B=\left(-v_{1 r} \sin (\phi)+v_{1 q} \cos (\phi)\right) / 2
\end{array}\right.
$$

In both the cases, to plot the contours on the Smith chart, the obtained solutions in terms of voltage can be translated to impedances by denormalizing the voltage components and applying Ohm's law (the current components are known).

\section{B. Higher Harmonics Clipping Contours}

The normalized voltage waveform is written as

$$
v(\theta)=1-\sum_{n=1}^{N}\left(v_{n r} \cos (n \theta)+v_{n q} \sin (n \theta)\right)
$$

and its derivative is

$$
d v(\theta) / d \theta=\sum_{n=1}^{N} n\left(v_{n r} \sin (n \theta)-v_{n q} \cos (n \theta)\right) .
$$

Also in this case, all harmonic voltages will be known, except the ones at the $k$ th harmonic. Repeating the calculations done for the second harmonic case, we find

$$
\left\{\begin{array}{l}
v_{k r}=(1-A) \cos (k \phi)+B \sin (k \phi) \\
v_{k q}=(1-A) \sin (k \phi)-B \cos (k \phi)
\end{array}\right.
$$

where

$$
\begin{aligned}
& A=\sum_{n=1, n \neq k}^{N}\left(v_{n r} \cos (n \phi)+v_{n q} \sin (n \phi)\right) \\
& B=\sum_{n=1, n \neq k}^{N} n\left(-v_{n r} \sin (n \phi)+v_{n q} \sin (n \phi)\right) / k
\end{aligned}
$$




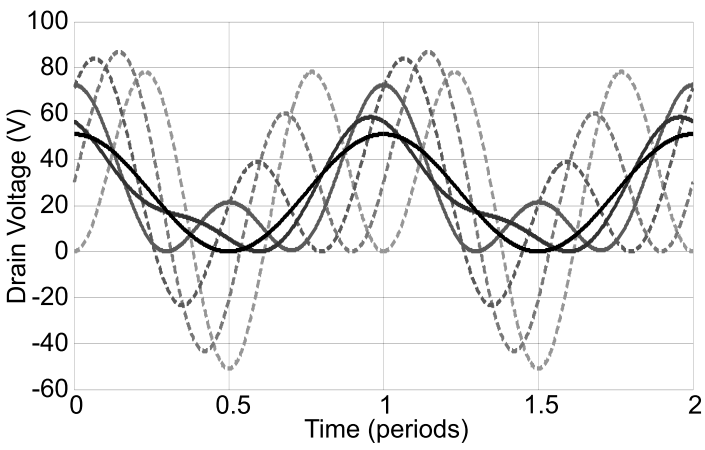

Fig. 1. Voltage drain waveforms for second harmonic clipping solutions for $Z_{1}=R_{\mathrm{opt}}=50 \Omega$. Input harmonics and higher output harmonics shortcircuited. Valid solutions (solid traces) and invalid solutions (dashed traces).

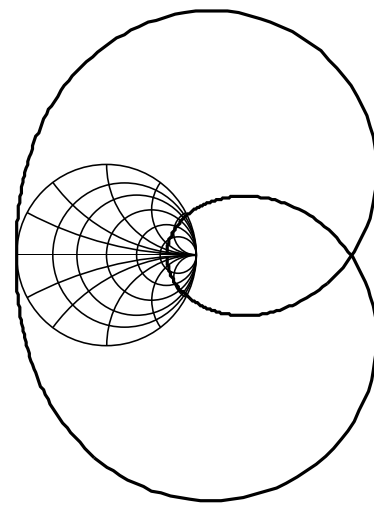

(a)

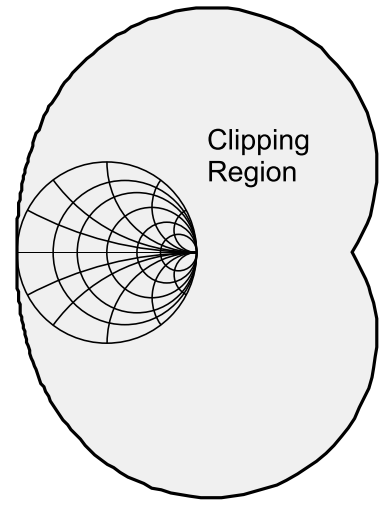

(b)
Fig. 2. Second harmonic clipping contours for fundamental load $Z_{1}=$ $R_{\mathrm{opt}}=50 \Omega$. Input harmonics and higher output harmonics are shortcircuited. (a) Including and (b) excluding invalid solutions.

\section{Example and Considerations}

To plot the clipping contours, apart from the load conditions at the non- $k$ th harmonic, four device parameters are needed. The drain current bias $\left(I_{\mathrm{DQ}}\right)$ and maximum $\left(I_{\mathrm{MAX}}\right)$ are used to determine the current Fourier components, while the drain voltage bias $\left(V_{\mathrm{DD}}\right)$ and minimum $\left(V_{\mathrm{min}}\right)$ are used to normalize and denormalize the voltage components.

As an example, a device with $I_{\mathrm{MAX}}=1 \mathrm{~A}, I_{\mathrm{DQ}}=$ $50 \mathrm{~mA}, V_{\mathrm{DD}}=28 \mathrm{~V}$, and $V_{\min }=2.5 \mathrm{~V}$ is considered. The fundamental optimum load results of $R_{\text {opt }} \simeq 50 \Omega$. Fig. 1 shows the drain voltage waveforms related to some possible solution for the second harmonic clipping contours when the fundamental load is $Z_{1}=R_{\mathrm{opt}}$, and $Z_{\mathrm{n}}=0$ for $n \geq 3$. It has to be noted that this new formulation also provides invalid solutions where the identified minimum is a local minimum and there is actually another absolute minimum lower than 0 . For this reason, it is necessary to execute a sweep of the solutions to exclude invalid ones. However, this operation can be optimized in a software tool, and has a negligible impact on its usability. Fig. 2 compares the second harmonic clipping contour in the cases where the invalid solutions are included and not included.

Although the proposed method is able to predict the clipping contours at any harmonic, throughout this paper the focus is on the second harmonic clipping contours, since they are the most

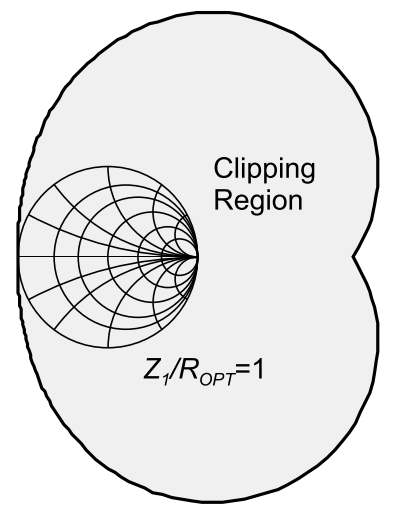

(a)

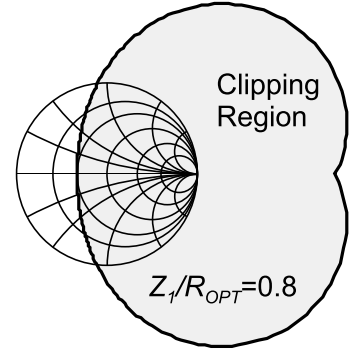

(b)
Fig. 3. Second harmonic clipping contours for fundamental load (a) $Z_{1}=R_{\mathrm{opt}}=50 \Omega$ and (b) $Z_{1}=0.8 R_{\mathrm{opt}}=40 \Omega$. Input harmonics and higher output harmonics are short-circuited.

important from a design perspective. In fact, in a broadband design, the second harmonic frequency can result very close, or even inside, the range of fundamental frequencies, thus making it more difficult, or even not feasible, to obtain a purely reactive second harmonic load. For higher harmonics this situation rarely verifies, especially in telecom applications. However, the third harmonic load is considered for its importance in moving the position of the second harmonic clipping contours.

To recall the utility of the harmonic clipping contours method from a design perspective, Fig. 3 compares the second harmonic clipping contours for different fundamental loads. In Fig. 3(a), where the fundamental load is set at the optimum for power, the gray area representing the clipping second harmonic loads covers the whole Smith chart, except the single point at a short circuit. By reducing the fundamental load as in Fig. 3(b), the clipping region shrinks relaxing the short circuit constraint on the second harmonic termination, which now has a larger design space. This means that linear operation over a broad bandwidth can be achieved by a controlled reduction of the power targets. It is also to be noted that a reduction of input drive can lead to a very similar extension of the nonclipping region, but in that case the corresponding power reduction would be quadratic instead of linear. A similar behavior can be observed when considering a fundamental load for class $\mathbf{J}$ operation, i.e., $Z_{1}=(1+j) R_{\mathrm{opt}}=(50+j 50) \Omega$ (see Fig. 4) [7], [17]. Also, in this case the reduction of the real part of the fundamental load "opens" a nonclipping region in the Smith chart.

Fig. 5 shows the second harmonic clipping contours, considering the example device introduced earlier, and third harmonic load at the optimum for class F. In Fig. 5(a), the fundamental load is set at $Z_{1}=1.15 R_{\mathrm{opt}}=57.5 \Omega$, corresponding to the standard class-F power increase with respect to class B; the whole Smith chart is included in the clipping region, except for the short circuit, as expected from class-F theory. Fig. 5(b) shows the case with $Z_{1}=R_{\mathrm{opt}}=$ $50 \Omega$ : thanks to the tuning of the third harmonic load, a larger part of the Smith chart becomes an available design space for the second harmonic without current clipping, but without 


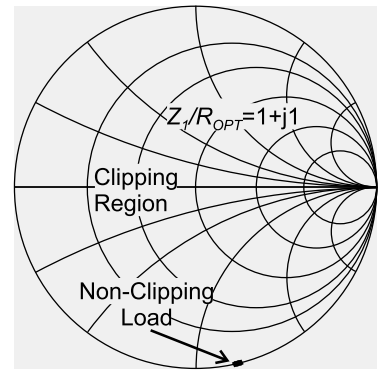

(a)

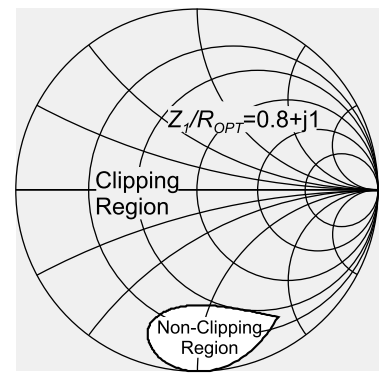

(b)
Fig. 4. Second harmonic clipping contours for fundamental load (a) $Z_{1}=$ $R_{\mathrm{opt}}+j R_{\mathrm{opt}}=(50+j 50) \Omega$ and (b) $Z_{1}=0.8 R_{\mathrm{opt}}+j R_{\mathrm{opt}}=(40+j 50) \Omega$. Input harmonics and higher output harmonics are short-circuited.

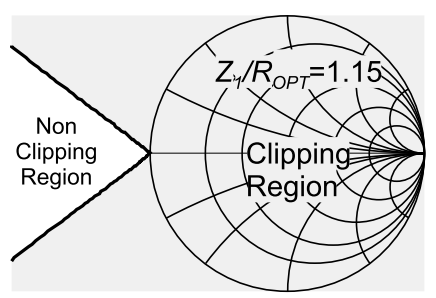

(a)

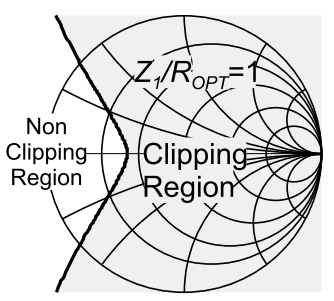

(b)
Fig. 5. Second harmonic clipping contours for fundamental load (a) $Z_{1}=$ $1.15 R_{\mathrm{opt}}=57.5 \Omega$ and (b) $Z_{1}=R_{\mathrm{opt}}=50 \Omega$. Third harmonic load for class F. Input harmonics and higher output harmonics are short-circuited.

the need to reduce the fundamental load below $R_{\text {opt }}$. This result is a clear indication that introducing higher harmonics in the computation of clipping contours opens new degrees of freedom for the design of PAs, thus allowing more freedom in the positioning of the second harmonic load without compromising the output power target.

\section{EXPERIMENTAL VERIFICATION}

\section{A. Method}

The clipping contours have been verified for the first time with a comprehensive characterization using the setup portrayed in Fig. 6, a three-harmonics active sourceand load-pull system with waveform measurement capability [18], [19]. A probe station is used for the die characterization. The system is controlled by a software suite developed by Mesuro Ltd. The nonlinear vector network analyzer (N5242A) collects the power waves from the external directional couplers and reconstructs the current/voltage waveforms thanks to the stable phase reference provided by a comb-generator. Vector, absolute amplitude, and phase calibration are performed using the analyzer utility. The load termination at fundamental, second, and third harmonics, and the source termination at second and third harmonics are controlled by adjusting the phase and amplitude of additional RF sources (Agilent ESG and PSG families). The signals are then multiplexed and injected into the device ports. The RF sources are synchronized with a $10-\mathrm{MHz}$ reference signal.

Load-pull is used to sweep the second harmonic load on a grid, in order to identify the clipping contours, and to position the fundamental and third harmonic loads at different

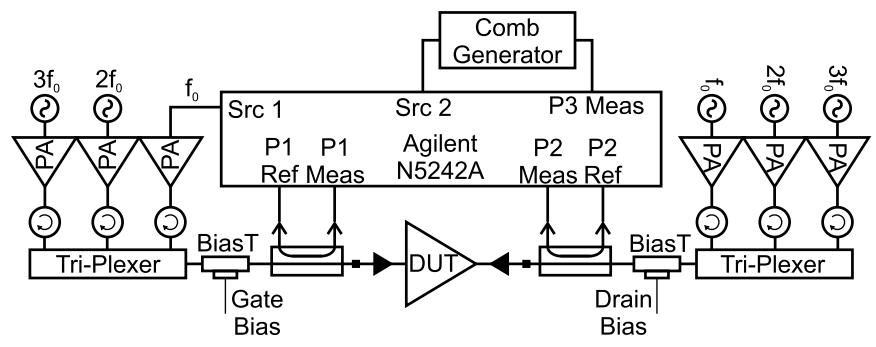

(a)

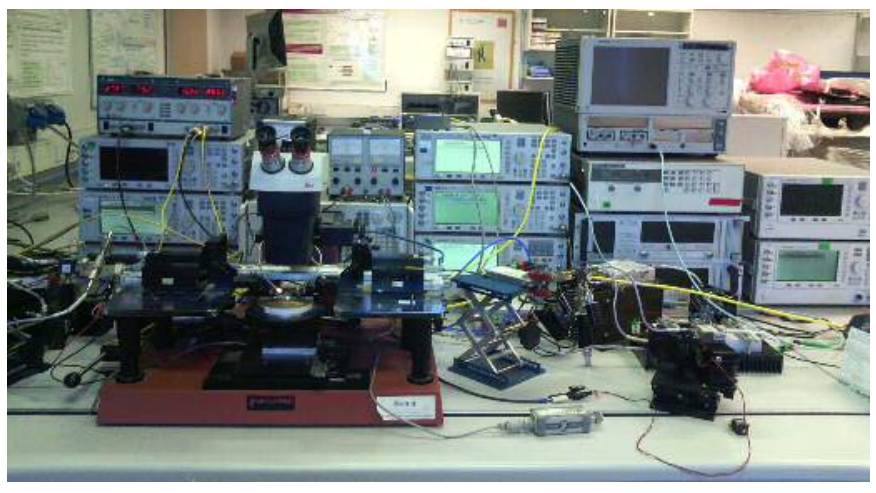

(b)

Fig. 6. (a) Block diagram and (b) picture of the source- and load-pull measurement system.

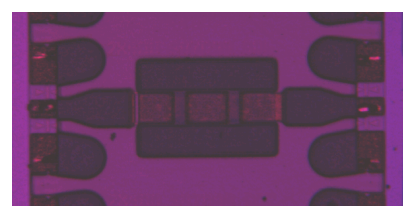

(a)

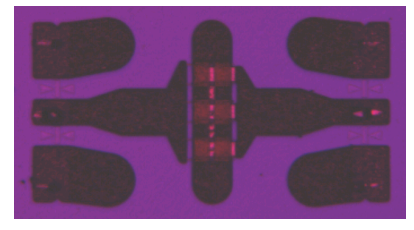

(b)
Fig. 7. Microscope pictures of (a) $2 \times 400 \mu \mathrm{m}$ and (b) $6 \times 80 \mu \mathrm{m}$ devices.

impedances. The effect of higher harmonics loaded with $50 \Omega$ instead of a short circuit is negligible, since it is mitigated by the output capacitance of the device.

Harmonic source-pull is used to short circuit the input harmonics at the gate reference plane of the device, in order to suppress the harmonic voltage components generated by the nonlinear input capacitance and to minimize the role of drainto-gate feedback. As a consequence, a drain current shaping similar to the ideal truncated sinewave can be guaranteed, and the risk of instability generated by the harmonic-load pull exploring extreme load conditions can be minimized.

The selected fundamental frequency for the characterization is $2 \mathrm{GHz}$, which is the maximum frequency manageable with the available setup, and that represents a reasonable choice when considering mobile telecom applications.

The following approach is followed in the characterization:

1) fundamental load-pull, with shorted source/load harmonics, performed to identify the extrinsic optimum load $Z_{\text {opt }}$ for output power;

2) estimation of output capacitance $C_{\text {OUT }}$ for waveform de-embedding at the intrinsic current generator plane;

3) identification, on a power sweep in optimum load conditions, of drive level and of associated $I_{\mathrm{MAX}}$ and $V_{\min }$; 
TABLE I

Fundamental LoAd-Pull: MEASURed Parameters WITH SHORTED HARMONICS

\begin{tabular}{|c|c|c|c|c|c|}
\hline Device & $Z_{\text {opt }}$ & $P_{\mathrm{SAT}}$ & $P A E_{\mathrm{SAT}}$ & $R_{\text {out }}$ & $C_{\text {OUT }}$ \\
\hline $2 \times 400 \mu \mathrm{m}$ & $73+\mathrm{j} 35 \Omega$ & $36 \mathrm{dBm}$ & $60 \%$ & $90 \Omega$ & $0.43 \mathrm{pF}$ \\
\hline $6 \mathrm{x} 80 \mu \mathrm{m}$ & $172+\mathrm{j} 63 \Omega$ & $36 \mathrm{dBm}$ & $60 \%$ & $195 \Omega$ & $0.15 \mathrm{pF}$ \\
\hline
\end{tabular}

TABLE II

Measured Key Parameters for the Prediction OF ClipPing CONTOURS

\begin{tabular}{|c|c|c|c|c|}
\hline Device & $I_{\mathrm{MAX}}$ & $I_{\mathrm{DQ}}$ & $V_{\mathrm{DD}}$ & $V_{\min }$ \\
\hline $2 \times 400 \mu \mathrm{m}$ & $590 \mathrm{~mA}$ & $31 \mathrm{~mA}$ & $28 \mathrm{~V}$ & $6.4 \mathrm{~V}$ \\
\hline $6 \times 80 \mu \mathrm{m}$ & $355 \mathrm{~mA}$ & $18 \mathrm{~mA}$ & $50 \mathrm{~V}$ & $15 \mathrm{~V}$ \\
\hline
\end{tabular}

4) second harmonic load sweep, at the selected drive level and with the other harmonic terminations set at arbitrary values;

5) tracing of minimum drain voltage contours versus second harmonic load, at which $\min \left\{v_{\mathrm{ds}}(t)\right\}=V_{\min }$;

6) comparison with predicted contours at the intrinsic generator plane.

\section{B. Devices}

Two GaN HEMT on SiC substrate devices are considered for the validation of the clipping contours theory; a $2 \times 400 \mu \mathrm{m}$ device based on $0.5-\mu \mathrm{m}$ technology and biased at $28-\mathrm{V}$ drain voltage, and a $6 \times 80 \mu \mathrm{m}$ device based on $0.25-\mu \mathrm{m}$ technology and biased at $50 \mathrm{~V}$. Fig. 7 shows the microscope pictures of the characterized devices.

Fundamental load-pull, with other harmonic terminations short-circuited, has been performed on both devices and the main results are summarized in Table I. The $R_{\mathrm{opt}}$ and $C_{\mathrm{OUT}}$ values are identified as $1 / Z_{\mathrm{opt}}=1 / R_{\mathrm{opt}}-j \omega C_{\text {OUT }}$.

A power sweep into a fundamental optimum load allows the identification of the key parameters for the calculation of the clipping contours and of the successive characterization steps, see Table II and Fig. 8. The $6 \times 80 \mu \mathrm{m}$ device starts exhibiting clipping at a significantly higher minimum drain voltage with respect to the $2 \times 400 \mu \mathrm{m}$ device. This is due to knee walk-out occurring when biasing at $50 \mathrm{~V}$.

The drain waveforms measured at the selected drive level are shown in Fig. 9; it can be noted that the drain current is just at the onset of clipping, thus providing a condition where strong nonlinearity is still negligible, but with reasonable output power and efficiency. We select the drive level according to this qualitative waveform observation, instead of relying on gain compression figures, which are usually misleading when using $\mathrm{GaN}$ devices characterized by evident soft-compression. Furthermore, in this drive level condition, the measured current harmonic components are in accordance with the current components predicted assuming a truncated sinusoid.

\section{Clipping Contours Results}

The second harmonic clipping contours for the two devices are calculated and measured, at the intrinsic current generator plane, considering three families of waveforms: class $A B$, class $\mathrm{J}$, and class $\mathrm{F}$.

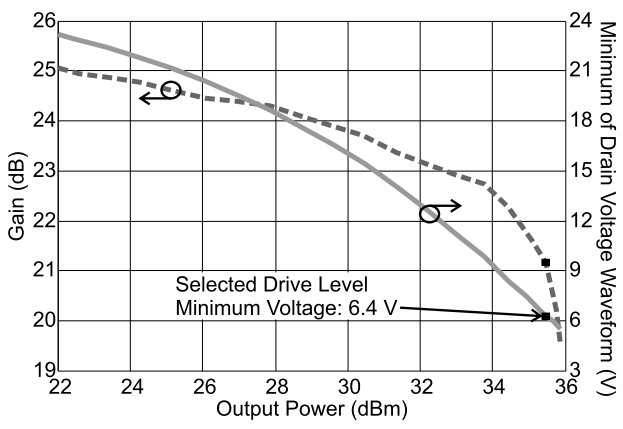

(a)

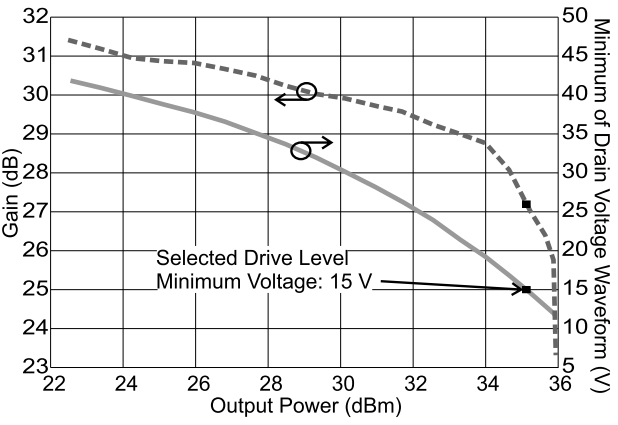

(b)

Fig. 8. Measured gain (dashed trace) and minimum drain voltage (solid trace) versus output power for (a) $2 \times 400 \mu \mathrm{m}$ and (b) $6 \times 80 \mu \mathrm{m}$ device, respectively. The drive level selected for the clipping contours measurement, with the corresponding minimum voltage, is highlighted.

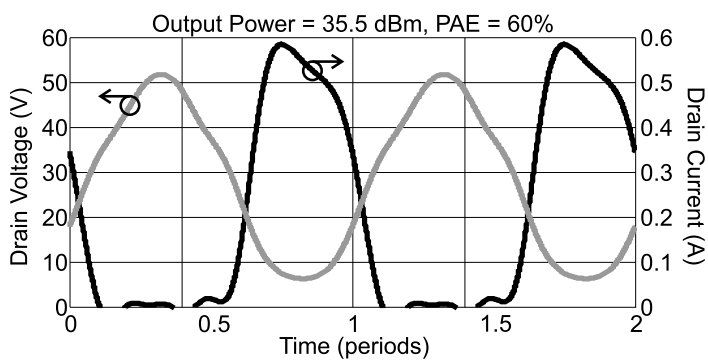

(a)

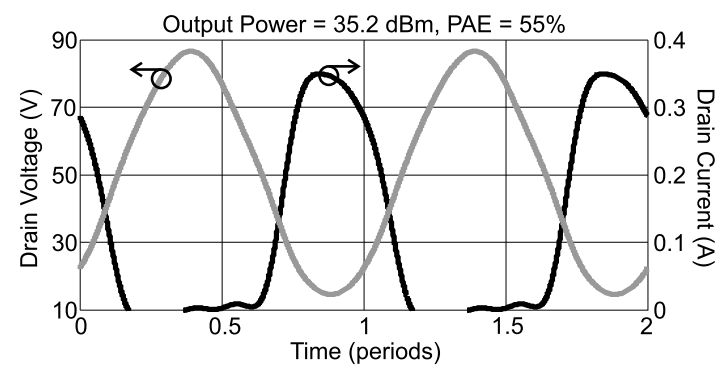

(b)

Fig. 9. Measured drain voltage (gray) and current (black) waveforms for (a) $2 \times 400 \mu \mathrm{m}$ and (b) $6 \times 80 \mu \mathrm{m}$ device, respectively, at the selected drive level. The corresponding output power and PAE are indicated.

For the class $\mathrm{AB}$ case the intrinsic fundamental load $Z_{1}$ is set on a purely real value, and the third harmonic load is shortcircuited. The trend of the second harmonic clipping contours is observed for $Z_{1}$ at $R_{\text {opt }}$ (maximum output power load), $0.9 R_{\mathrm{opt}}$, and $0.8 R_{\mathrm{opt}}$ (corresponding to an output power reduction of $1 \mathrm{~dB}$ ). The predicted and measured contours for the two devices are shown in Fig. 10. The second harmonic reflection 

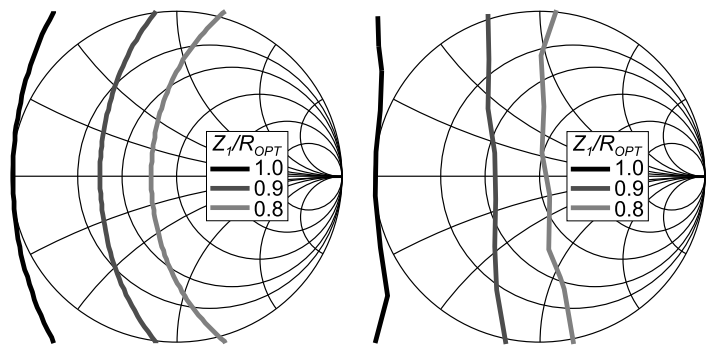

(a)
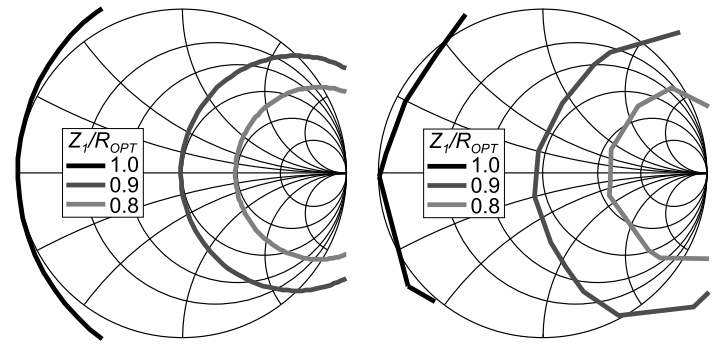

(b)

Fig. 10. Calculated (left) and measured (right) second harmonic clipping contours for (a) $2 \times 400 \mu \mathrm{m}$ and (b) $6 \times 80 \mu \mathrm{m}$ device, respectively, when varying the fundamental load $Z_{1}$ on the real axis. The third harmonic load $Z_{3}$ has been short-circuited.
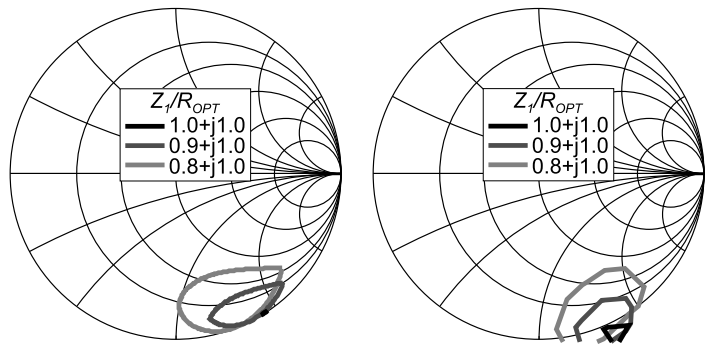

(a)
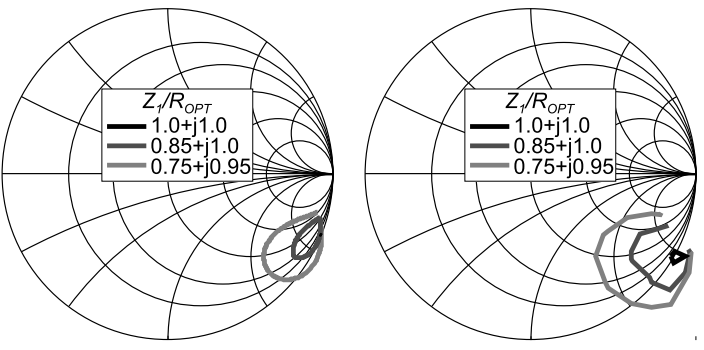

(b)

Fig. 11. Calculated (left) and measured (right) second harmonic clipping contours for (a) $2 \times 400 \mu \mathrm{m}$ and (b) $6 \times 80 \mu \mathrm{m}$ device, respectively, when varying the fundamental load $Z_{1}$ around the class $\mathrm{J}$ optimum. The third harmonic load $Z_{3}$ has been short-circuited.

coefficient $\Gamma_{2}=\left(\left(Z_{2}-50\right) /\left(Z_{2}+50\right)\right)$ was swept on a uniform $8 \times 8$ (real $\times$ imaginary) rectangular grid, between -1 and 1 . Good agreement between measured and estimated contours can be observed for both the devices. In fact, not only is the trend versus fundamental load confirmed, but also the actual position of the contours is predicted satisfactorily. For the class $\mathrm{J}$ case, the intrinsic fundamental load $Z_{1}$ is set around the optimum for class $\mathrm{J}, Z_{1}=R_{\mathrm{opt}}+j R_{\mathrm{opt}}$, and the calculated and measured contours are shown in Fig. 11. The second harmonic reflection coefficient $\Gamma_{2}$ was swept on an $8 \times 8$ rectangular uniform grid, then a second sweep on a

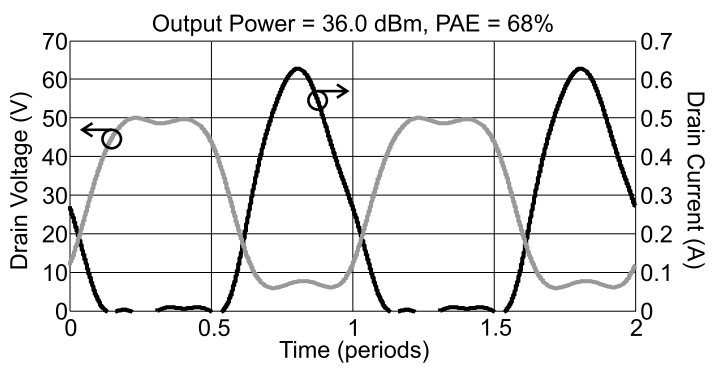

(a)

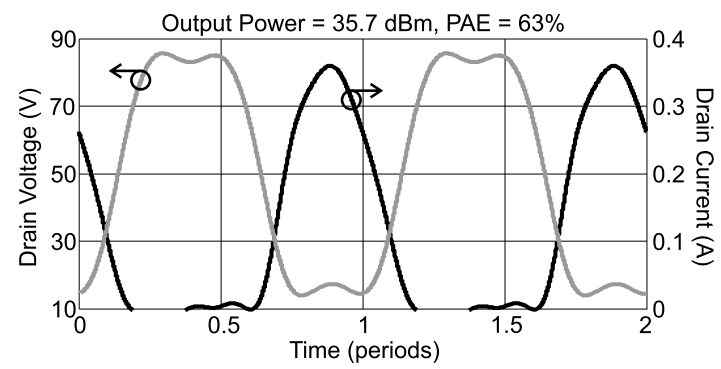

(b)

Fig. 12. Measured drain voltage (gray) and current (black) waveforms for (a) $2 \times 400 \mu \mathrm{m}$ and (b) $6 \times 80 \mu \mathrm{m}$ device, respectively, at the selected drive level for class-F loading condition. The corresponding output power and PAE are indicated.
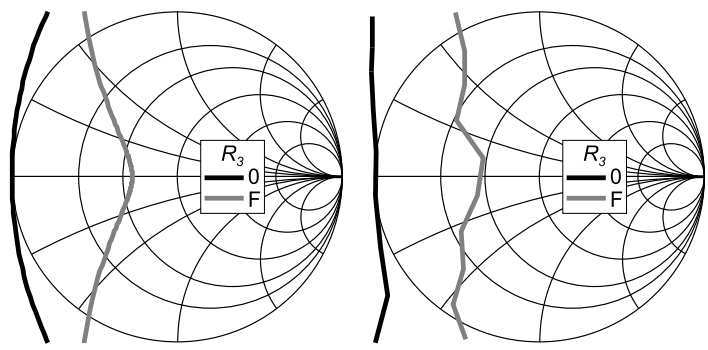

(a)
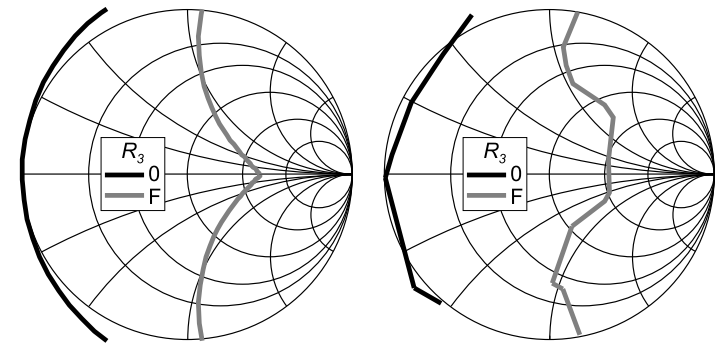

(b)

Fig. 13. Calculated (left) and measured (right) second harmonic clipping contours for (a) $2 \times 400 \mu \mathrm{m}$ (b) and $6 \times 80 \mu \mathrm{m}$ device, respectively, when $Z_{3}$ is at a short circuit (black) or at the optimum for class-F (gray). $Z_{1}=R_{\mathrm{opt}}$.

smaller area with $7 \times 7$ grid was used to refine the contour. Also in this case, good agreement between measured and estimated contours is achieved for both the devices.

In order to verify the trend of the second harmonic clipping contours when the third harmonic load is tuned, an initial tuning of the third harmonic load has been performed to find the proper termination that delivers a class-F voltage waveform. The resulting measured waveforms, which correspond to a $Z_{1}=1.15 R_{\text {opt }}$ at the selected drive level, are shown in Fig. 12.

In the next step, the third harmonic load is kept at the chosen value, and the second harmonic load is swept to determine the 
clipping contour. Fig. 13 compares the predicted and measured contours for a short-circuited third harmonic load and at the optimum for class $\mathrm{F}$, while maintaining $Z_{1}=R_{\text {opt }}$.

It is interesting to notice how the nonclipping region for the second harmonic can be extended through tuning of the third harmonic load instead of the fundamental load, without reducing the power with respect to class $\mathrm{AB}$ condition. This result highlights the importance of having extended the clipping contour theory beyond the second harmonic, and it opens new degrees of freedom in the design of broadband linear PAs.

\section{CONCLUSION}

A new formulation for determining harmonic clipping contours for high-frequency power devices has been presented in this paper. This new approach allows the inclusion of an arbitrary number of harmonics for the clipping contour prediction. The second harmonic clipping contours are thoroughly verified for the first time against load-pull measurements of two different GaN HEMTs, and very good agreement results from this comparison. Moreover, it has been demonstrated that the tuning of the third harmonic load permits the extension of the second harmonic design space without output power reduction, thus providing the designers with a powerful tool for the systematic design of linear PAs.

\section{ACKNOWLEDGMENT}

The authors would like to thank Dr. R. Saini for help in setting the characterization setup.

\section{REFERENCES}

[1] F. H. Raab et al., "Power amplifiers and transmitters for RF and microwave," IEEE Trans. Microw. Theory Techn., vol. 50, no. 3, pp. 814-826, Mar. 2002.

[2] F. H. Raab, "Maximum efficiency and output of class-F power amplifiers," IEEE Trans. Microw. Theory Techn., vol. 49, no. 6, pp. 1162-1166, Jun. 2001.

[3] P. Colantonio, F. Giannini, E. Limiti, and V. Teppati, "An approach to harmonic load- and source-pull measurements for high-efficiency PA design," IEEE Trans. Microw. Theory Techn., vol. 52, no. 1, pp. 191-198, Jan. 2004.

[4] P. Colantonio, F. Giannini, R. Giofre, E. Limiti, and A. Nanni, "Power amplifier design strategy to null IMD asymmetry," in Proc. 36th Eur. Microw. Conf. (EuMC), Sep. 2006, pp. 1304-1307.

[5] P. Colantonio, F. Giannini, R. Giofre, and L. Piazzon, "Simultaneous dual-band high efficiency harmonic tuned power amplifier in $\mathrm{GaN}$ technology," in Proc. Eur. Microw. Integr. Circuit Conf. (EuMIC), Oct. 2007, pp. $127-130$.

[6] M. Roberg and Z. Popovic, "Analysis of high-efficiency power amplifiers with arbitrary output harmonic terminations," IEEE Trans. Microw. Theory Techn., vol. 59, no. 8, pp. 2037-2048, Aug. 2011.

[7] S. C. Cripps, P. J. Tasker, A. L. Clarke, J. Lees, and J. Benedikt, "On the continuity of high efficiency modes in linear RF power amplifiers," IEEE Microw. Wireless Compon. Lett., vol. 19, no. 10, pp. 665-667, Oct. 2009.

[8] V. Carrubba et al., "The continuous class-F mode power amplifier," in Proc. Eur. Microw. Integr. Circuits Conf. (EuMIC), Sep. 2010, pp. $432-435$.

[9] S. Rezaei, L. Belostotski, M. Helaoui, and F. M. Ghannouchi, "Harmonically tuned continuous class-C operation mode for power amplifier applications," IEEE Trans. Microw. Theory Techn., vol. 62, no. 12, pp. 3017-3027, Dec. 2014.

[10] M. Ozen et al., "Wideband and efficient watt-level SiGe BiCMOS switching mode power amplifier using continuous class-E modes theory," in Proc. IEEE Radio Freq. Integr. Circuits Symp., Jun. 2014, pp. $243-246$.
[11] B. M. Merrick, J. B. King, and T. J. Brazil, "The continuous harmonictuned power amplifier," IEEE Microw. Wireless Compon. Lett., vol. 25 , no. 11, pp. 736-738, Nov. 2015.

[12] P. Wright, J. Lees, J. Benedikt, P. Tasker, and S. Cripps, "A methodology for realizing high efficiency class-J in a linear and broadband PA," IEEE Trans. Microw. Theory Techn., vol. 57, no. 12, pp. 3196-3204, Dec. 2009.

[13] T. Canning, P. Tasker, and S. Cripps, "Continuous mode power amplifier design using harmonic clipping contours: Theory and practice," IEEE Trans. Microw. Theory Techn., vol. 62, no. 1, pp. 100-110, Jan. 2014.

[14] C. Friesicke, R. Quay, and A. F. Jacob, "The resistive-reactive class-J power amplifier mode," IEEE Microw. Wireless Compon. Lett., vol. 25, no. 10 , pp. 666-668, Oct. 2015.

[15] R. Quaglia and S. Cripps, "Harmonic clipping contours: Numerical computation and extension to higher harmonics," in Proc. Eur. Microw. Conf. (EuMC), Oct. 2016, pp. 1-4.

[16] P. Colantonio, F. Giannini, and E. Limiti, High Efficiency $R F$ and Microwave Solid State Power Amplifiers. Hoboken, NJ, USA: Wiley, 2009.

[17] F. H. Raab, "Class-E, class-C, and class-F power amplifiers based upon a finite number of harmonics," IEEE Trans. Microw. Theory Techn., vol. 49, no. 8, pp. 1462-1468, Aug. 2001.

[18] V. Camarchia, V. Teppati, S. Corbellini, and M. Pirola, "Microwave measurements. Part II-Non-linear measurements," IEEE Instrum. Meas. Mag., vol. 10, no. 3, pp. 34-39, Jun. 2007.

[19] R. Quaglia, D. J. Shepphard, and S. Cripps, "A reappraisal of optimum output matching conditions in microwave power transistors," IEEE Trans. Microw. Theory Techn., vol. 65, no. 3, pp. 838-845, Mar. 2017.

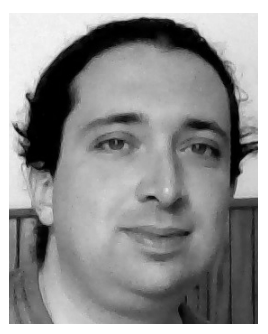

Roberto Quaglia (M'13) was born in Casale Monferrato, Italy, in 1984. He received the bachelor's degree (cum laude) in electronic engineering and $\mathrm{Ph} . \mathrm{D}$. degree in electronic devices from the Politecnico di Torino, Turin, Italy, in 2008 and 2012, respectively.

His current research interests include the design, modeling, and predistortion of high-efficiency MMIC power amplifiers.

Dr. Quaglia is currently a European Union Marie Skłodowska Curie Fellow. He was a recipient of the 2009 Young Graduated Research Fellowship presented by the GAAS Association.

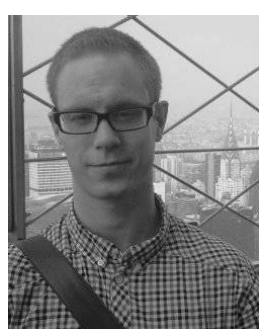

James J. Bell received the bachelor's and Ph.D. degrees from Cardiff University, Wales, U.K., in 2009 and 2013, respectively.

He was with the Centre for High Frequency Engineering, Cardiff University, where he was involved in the development of nonlinear measurement systems, the Cardiff model, and high-efficiency power amplifier design. He is currently the NI Lecturer with Cardiff University, where he is involved in behavioral modeling concepts, intelligent measurement systems, and broadband power amplifier design.

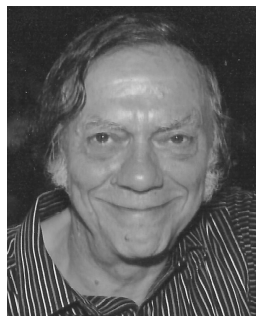

Steve Cripps (M'81-SM'90-F'11-LF'16) received the bachelor's, master's, and Ph.D. degrees from Cambridge University, Cambridge, U.K.

$\mathrm{He}$ was with the high frequency ("microwave") electronics industry, both in the U.K. and in the USA, where he was a Designer, a Manager, and an Independent Consultant. He is currently a Distinguished Research Professor with Cardiff University, Wales, U.K. He has authored several books on RF power amplifiers.

Prof. Cripps was a recipient of the 2008 IEEE Microwave Applications Award and the 2015 Microwave Prize. 